\title{
TV/Series
}

Hors séries 1 | 2016

Lost: (re)garder l'île

\section{Lost Experience : Statuts d'Emerson dans la série Lost}

Guillaume Dulong

\section{(2) OpenEdition \\ Journals}

Electronic version

URL: http://journals.openedition.org/tvseries/2207

DOI: $10.4000 /$ tvseries.2207

ISSN: 2266-0909

\section{Publisher}

GRIC - Groupe de recherche Identités et Cultures

Electronic reference

Guillaume Dulong, "Lost Experience : Statuts d'Emerson dans la série Lost », TV/Series [Online], Hors séries 1 | 2016, Online since 16 August 2018, connection on 05 May 2019. URL : http:// journals.openedition.org/tvseries/2207; DOI : 10.4000/tvseries.2207

This text was automatically generated on 5 May 2019.

\section{cc) (i) $\Theta$}

TV/Series est mis à disposition selon les termes de la licence Creative Commons Attribution - Pas d'Utilisation Commerciale - Pas de Modification 4.0 International. 


\title{
Lost Experience : Statuts d'Emerson dans la série Lost
}

\author{
Guillaume Dulong
}

Où nous trouvons-nous? Dans une série dont nous ne connaissons pas les extrémités, et dont nous croyons qu'elle n'en a point ${ }^{1}$.

1 Lost présente nombre de personnages homonymes de "grands hommes» qui se rencontrent voire s'affrontent sur l'île. En cela le récit reprend le genre de l'utopie et les thèmes de la société idéale, voire secrète/sacrée, et de son dérivé antonyme, la dystopie vue la quantité de morts, souvent stupides, dans les rangs de ces protagonistes à noms de personnalités - dans la lignée du Meilleur des Mondes d'Aldous Huxley qui mobilisait aussi ce trope de l'homonymie. Néanmoins, ce qui nous semble plus original dans l'adoption de ce procédé par Cuse et Lindelof est cette remarque que formule Jeremy Barris dans son article "Lost and the question of life after birth »: "What's more, the character Ben Linus is played by the actor Michael Emerson. Coincidence? Or has the island's electromagnetic field burst free of the writer's control and now started to draw our own world into its reality? "2

2 En effet l'acteur Michael Emerson qui joue le rôle de Benjamin Linus, l'un des principaux antagonistes du récit qui finira par garder l'île aux côtés de Hugo (Jorge Garcia), est luimême l'homonyme d'un philosophe américain, Ralph Waldo Emerson (1803-1882). Celuici est le chef de file du courant de pensée transcendantaliste, apologue d'une « confiance en soi » (self reliance) métaphysique comme aversion d'une tendance mimétique aliénante, le « conformisme ». "Croire en votre propre pensée, croire que ce qui est vrai au plus secret de votre cœur est vrai pour tous les hommes - là est le génie. $»^{3}$ Cette « confiance en soi » est souvent hâtivement associée à un dualisme économico-politique libéral opposant originalité solitaire, d'un côté, et uniformité du «troupeau », de l'autre, ou bien amour propre individuel et " conformisme » social ${ }^{4}$. Or, plus qu'un thème, ce sens et ce contresens de la confiance en soi seraient un ressort même de l'écriture et de la réception de l'œuvre d'Emerson interrogeant par là même l'identité de l'auteur et du lecteur, selon Cavell. 
Quelle signification peut alors avoir une telle référence dans Lost? En est-ce d'ailleurs seulement une, dans la mesure où elle concerne un élément du dispositif réel d'énonciation (l'état civil d'un acteur) et non pas l'énoncé fictif, la diégèse elle-même? Tout est une question de confiance, serions-nous enclin à dire. Quoique probable, c'est en courant le risque de considérer cette homonymie patronymique réelle comme un signe, c'est en courant le risque de prendre nos désirs pour des réalités et de se recueillir sur ceux-ci à travers notre réception des images que, nous, téléspectateurs, pourrons rétrospectivement les rendre signifiants.

4 «Le risque de se livrer à l'inessentiel est lui-même essentiel. » écrivait Blanchot, et sans doute avons-nous là, une illustration exemplaire du défi de ce que nous nommions précédemment, l'oscillation souterraine de Lost entre le Cygne et la Perle ${ }^{5}$. Si nous faisons retour sur la saison 2, nous constatons qu'il y a deux hôtes-otages du bunker : Desmond David Hume (Henry Ian Cusick) et un autre qui se fait appeler Henry Gale (Michael Emerson). À partir de ce constat ne pourrions-nous pas conjecturer que le cœur de la contamination fictionnelle du réel derrière tout l'appareil de marketing viral de la Lost Experience amorcé dans ladite saison (le vrai faux site Hanso, le livre Bad Twin de «Gary Troup » sur Widmore et son double...) eût été de présenter non pas un personnage, mais plutôt un acteur à nom de philosophe (auteur, entre autres, d'un essai intitulé Experience sur la perte de son fils et le deuil) jouant un personnage à pseudonyme? Qui plus est, il s'agit d'un personnage dont on ne connaît pas le nom à la fin de celle-ci. Les auteurs sontils allés jusqu'à convoquer le dispositif réel d'énonciation, en l'occurrence le nom des acteurs, dans la logique de l'histoire racontée? Dans ce cas quel serait le sens de cet incident de frontière entre réel et imaginaire?

5 Il est très improbable que Cuse et Lindelof n'ait pas vu de liens à tisser avec ce donné patronymique (ce nom du père) et son homonymie pour les faire travailler dans l'économie de la série télévisée et tracer le trajet du personnage, sa chute et sa rédemption, comme deux faces de la "confiance en soi»: amour propre d'un moi souverain, et lâcher prise comme retour au Soi. Ce qui nous encourage à suivre cette hypothèse est, qu'historiquement, Ralph Waldo Emerson était l'ami de Thomas Carlyle, homonyme du premier "sacrifice» de l'île, Boone (Ian Somerhalder), et était contemporain de Søren Kierkegaard. En outre, pour une série américaine qui brasse quelques références philosophiques, il est le seul philosophe des Etats-Unis - philosophe symptomatiquement considéré par Cavell comme un père fondateur refoulé de la pensée américaine ${ }^{6}$. Ajouterons-nous que le poème ouvrant l'essai Société et Solitude met en scène un barde dénommé Saïd, serviteur de la Nature et "coupe de perles», Saïd (Naveen Andrew) étant le nom du rescapé victime, puis bourreau, de Benjamin Linus à partir de la saison $4^{7}$ ? On nous répondrait sans doute, avec bon sens, qu'il s'agit d'une pétition de principe : il est facile de trouver ce que l'on cherche lorsqu'on veut avoir raison et que l'on sait déjà de quoi il s'agit. Toujours notre oscillation homme de foi/homme de science donc : s'agit-il d'une coïncidence ou est-ce totalement calculé ?

6 L'interrogation sur l'existence ou non d'une relation de sens entre ce personnage déchu et réhabilité dans la saison 6, et le penseur d'une psychologie transcendantale et d'un fatalisme quotidien, est ainsi indissociable d'une interrogation sur la manière dont la série présente son intelligence, c'est-à-dire la manière dont elle signifie intimement pour les téléspectateurs, les interprètes et les auteurs.

7 L'économie du récit est ainsi travaillée par le problème du " pour qui ? » afin de ménager un espace de relation, un entre nous. Pour qui l'auteur écrit-il le récit? Pour qui le 
téléspectateur le reçoit-il? Pour qui les personnages agissent-il et subissent-ils les péripéties? Pour qui est-ce que les auteurs se donnent à voir et donnent à voir les téléspectateurs en envisageant les personnages du récit? Pour qui est-ce que les téléspectateurs se donnent à voir et donnent à voir les auteurs en envisageant les personnages du récit? Et, corrélativement, avec ce que cette expression peut avoir de péjoratif en français, pour qui est-ce que les auteurs se prennent et prennent les téléspectateurs au moyen des acteurs? Pour qui est-ce que les téléspectateurs se prennent et prennent les auteurs au moyen des acteurs? Cette ambivalence du don et de l'appropriation dans l'économie de la représentation et de la reconnaissance reçoit ici un traitement interrogeant l'identité télévisuelle.

Essentiellement, voire pratiquement, le médium télévisuel représente l'acteur en personnage, différemment du théâtre, voire de romans historiquement inspirés; subséquemment l'opposition auteur/récepteur diffère également. Cette représentation sérielle est proche de celle que propose le cinéma quoique distincte. En raison du temps de diffusion, de sa longue durée et de ses coups de théâtre à répétitions, l'identité télévisuelle qu'elle esquisse paraît à la fois plus susceptible d'une défiguration et, paradoxalement, plus monotone dans sa multiplication des alias.

En mobilisant les analyses de Cavell dans The World Viewed, sur le type d'individuation associé au médium audiovisuel, nous montrerons qu'il n'est pas absurde que le nom d'Emerson soit signifiant pour comprendre le récit audiovisuel. Puis nous relierons cet examen à un problème travaillant l'écriture et la réception de la série : la compréhension $\mathrm{du}$ principe d'indétermination du réel à l'aune de l'opposition éthique, voire métaphysique, de la confiance et la défiance - problème qui nous occupait dans une étude précédente intitulée «La Promesse de Lost " parue dans cette même revue. Notre article prolongera donc le propos de cette dernière en mettant l'accent, à travers l'examen du procédé de l'homonymie, sur la thématique de la relation intersubjective, de communauté et d'intimité, entre les personnages dans le récit, mais aussi de la relation entre auteurs et téléspectateurs. Si ce n'est que dans le cadre d'une interrogation sur la possibilité pour l'homonymie du patronyme d'un acteur de faire sens au sein même d'une histoire fictive, nous questionnerons également la représentation de l'identité voire la subjectivité audiovisuelle et les conditions de sa reconnaissance.

\section{Alias}

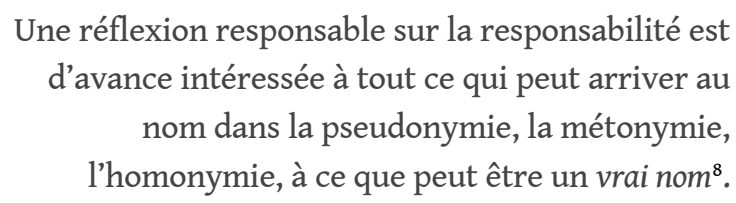

Comment le patronyme Emerson de l'acteur qui joue le rôle de Benjamin Linus pourrait-il faire sens pour comprendre le récit de Lost, au même titre que font sens les homonymies avec des personnalités historiques des personnages dans la série comme Locke, Rousseau, Austen, Lewis ou Farraday?

11 En théorie, il y a une différence entre des références que le téléspectateur peut reconnaître comme étant intentionnellement laissées par les auteurs et celles qui tiennent à des circonstances indépendantes de leurs volontés, ou considérées comme telle dans le contexte de communication qu'est la narration. À moins d'avoir une aptitude 
d'anticipation hors du commun, les showrunners étaient incapables de baptiser leur acteur - cela fut plus vraisemblablement le rôle institutionnel de ses parents - afin de le prendre dans une série qui serait tournée bien des années après. De surcroit, Damon Lindelof étant plus jeune que Michael Emerson cela rendrait l'opération a priori impossible... Toutefois le doute persiste : Emerson a passé un casting, il a donc été retenu sur une liste par une équipe en contact avec les auteurs qui ont pu réorienter l'écriture du récit pour exploiter ce kaïros, cette opportunité. Dans ce cas ces derniers n'auraient-ils pas dû lui conserver son patronyme dans la fiction afin que ladite référence historique soit manifeste?

12 Nous pouvons rétorquer que ce personnage apparait pour la première fois dans la saison 2 dans l'épisode « One of them » (S02E14) et que tout l'enjeu des épisodes où il est présent, comme otage des rescapés, est de déterminer son identité, c'est-à-dire s'il s'appelle bien Henry Gale ou s'il n'est pas un «Autre ». Lorsque ceux-ci découvrent que ce n'est qu'un pseudonyme, le personnage reste anonyme jusqu'à l'épisode final de ladite saison où il se fera reconnaitre comme le chef des Autres.

D'autre part, l'identité des personnages est sujette à variation voire à altération, un personnage peut se ressembler physiquement mais changer moralement et vice versa. C'est le problème de l'identité personnelle privée et publique, de l'identité personnelle comme phénomène psychologique et théorique ou comme phénomène pratique et social, et du rôle du langage dans leur opposition. Ce problème travaille la philosophie de David Hume dans Le Traité sur la Nature Humaine reprenant explicitement les réflexions des Essais sur l'Entendement Humain de John Locke ${ }^{9}$. L'identité subjective, en théorie, relève de la croyance et de l'habitude plus que d'un véritable savoir : à force d'associer des idées de sensation et de réflexion ressemblantes et contiguës, mais distinctes dans l'espace, et surtout dans le temps, nous croyons qu'il existe une identité profonde, objective ou subjective, qui causerait ces ressemblances et contiguïtés d'idées. Je suis enclin à penser qu'un même "moi » combine ces pensées par une opération psychique spontanée. Pourtant, théoriquement, rien ne m'assure que ces impressions, fondamentalement diverses et variées, ne s'associent pas elles-mêmes, mécaniquement, produisant cette image d'unité stable conforme à la pérennité du cours impersonnel de ces associations. L'exercice et l'économie politique et poétique du langage, nous assignant nom propre et patronyme afin de prendre la parole et répondre, d'être responsables, accréditent plus qu'ils ne certifient, l'existence de cette identité subjective qui n'est au mieux qu'une hypothèse. Cependant l'exigence pour le groupe de se constituer lui-même une identité commune à travers des échanges sociaux stables, l'exigence de conformité, présente cette hypothèse théorique comme une nécessité morale, un impératif. Le paradoxe est donc que, grâce à la situation intermédiaire de l'exercice du langage entre théorie solitaire et pratique sociale, nous doutons que nous demeurions les "mêmes" psychologiquement, et tout à la fois exigeons socialement de demeurer les «mêmes ». Subséquemment la multiplication des pseudonymes et des homonymes dans la série qui mêle, en outre, références fictionnelles et historiques interroge donc le paradoxe de cette identité et responsabilité à mi-chemin entre le privé et le public.

Ainsi Benjamin Linus est un des trois noms du personnage joué par Michael Emerson. C'est, certes, le plus utilisé pour le désigner à partir de la saison 3, mais c'est vraisemblablement l'homonyme d'un personnage historique. Linus serait le nom du premier pape de l'église catholique après Pierre. Vus les références messianiques vétéro et néo testamentaires de la série, on pourrait comprendre le rapport de ce nom avec un 
personnage déchu comme étant une critique de l'Eglise et des institutions religieuses: celles-ci représentent un sentiment ou une intuition théologique qu'elles exploitent et subvertissent à leur propre profit. Que le Gardien de la Lumière de l'île, Jacob (Mark Pellegrino), soit assassiné par celui-là même qui prétend le représenter, signifierait que l'inspiration est pervertie par l'institution qui s'en réclame, celle-ci révélant sa propre vacuité à mesure qu'elle accroît son pouvoir dans le monde ${ }^{10}$. Subséquemment, on pourrait inférer que le récit nous avertit qu'il est logique que le téléspectateur qui entend le mieux se représenter Lost lui substitue "son" Lost, un récit au sens totalement maîtrisé, illustrant ainsi la paronymie italienne: "Traduttore traditore.», et cherche à l'imposer aux « Autres » : téléspectateurs et auteurs. Cependant comme nous l'avons dit, ce personnage se fait aussi appeler Henry Gale dans la saison 2, et James Moriarty, comme l'ennemi juré de Sherlock Holmes, le « Napoléon du crime », dans l'épisode «The Shape of things to come » (S04E09). À la rigueur, nous pourrions presque mentionner un quatrième nom, Workman, attribué par Hurley dans l'épisode « Tricia Tanaka is Dead » (S03E10), en voyant le cadavre de Roger Linus avec le bleu de son fils. Or Artisan (Workman en anglais) est le nom du héros, d'ascendance basque, du roman Bad Twin de Gary Troup/Laurence Shame, nom attribué à son arrière-grand-père à Ellis Island, à la suite d'un malentendu des autorités entre patronyme et profession.

Comme d'autres personnages sont des homonymes de personnalités historiques dans le récit, on pourrait arguer qu'il s'agit juste «d'accidents" pour eux aussi - ce qui est également peu probable. Qu'un personnage au départ anonyme dans la fiction soit considéré en fonction du patronyme de l'acteur et de son homonymie avec une personnalité historique peut être considéré comme une manière par laquelle le récit se propose de réfléchir sur l'identité personnelle, sa reconnaissance et son aliénation dans et par ses relations à autrui. Le recourt aux pseudonymes, hétéronymes voire homonymes est un ressort de l'écriture de l'œuvre de Kierkegaard dont l'influence nous semble prégnante dans la série. Il interrogerait ainsi la patronymie, l'autorité à l'œuvre sur l'œuvre et le lecteur, ce qui, selon Derrida, est à relier au fonds abrahamique de la littérature. L'acte d'écrire, tel qu'il est spécifiquement réfléchi par Kierkegaard dans Crainte et tremblement et La Reprise, et dans La lettre au père de Kafka, est un sacrifice impossible car toujours exigé mais déjà transgressé donc à se faire pardonner, oscillant entre le respect du nom du père et le désir de prendre femme ${ }^{11}$. Cette tension nous paraît travailler les « malades d'amour » aux troubles patronymiques de Lost et particulièrement Benjamin Linus/Emerson dont on peut supposer que l'attente finale hors de l'Église, attente probable d'une union avec Rousseau (Mira Furlan) grâce à Alex (Tania Raymonde), est aussi celle d'un au-delà du récit où seront réconciliées ces deux exigences.

Enfin, comme nous le faisions remarquer dans notre article précédent, et comme nous le développerons ensuite, cette indétermination est l'enjeu même de Lost, cette histoire sur l'incident dont on se demande s'il nous destiné ou non, qui peut en répondre et comment. Le téléspectateur est amené à s'interroger sur les conditions de reconnaissance de la responsabilité de l'acte de raconter, tant quant à l'auteur que quant à lui-même, et de s'interroger subséquemment sur ce qui est ou non intentionnel. Dès lors, ne parait-il pas invraisemblable qu'en nous montrant ce personnage les auteurs aient l'intention de nous montrer aussi l'acteur qui joue le personnage. Néanmoins, en nous faisant hésiter de la sorte, il nous semble que Cuse et Lindelof suggèrent que cette question de la responsabilité des showrunners, appelant à ce que les téléspectateurs en répondent à leur tour, n'est jamais totalement réductible à une justification intentionnelle déterminée par 
les termes d'un contrat de lecture déjà écrit où auteur et spectateur sont sur un strict pied d'égalité, où chacun sait déjà quoi exiger ou non du récit.

On nous objectera que pour appréhender l'histoire, nous sommes contraints de faire abstraction de l'identité civile des acteurs afin de voir leur personnage, sinon nous confondrions réel et imaginaire, l'homme et celui qu'il représente. Cette objection est valable, à la rigueur, pour le théâtre. En revanche, la série nous fait hésiter entre l'acteur et le rôle car cette hésitation est essentielle au médium audiovisuel. En d'autres termes, c'est ce que font les images cinématographiques et télévisuelles.

Comme l'écrit Cavell à la suite de Bazin et Panofsky, ces médias ne représentent pas le monde comme le font la peinture ou une pièce de théâtre ${ }^{12}$. Par leur nature, les images photographiques, voire électroniques pour la télévision, sont réelles, c'est-à-dire sont $d u$ réel : ce sont des objets, des effets, voire des parties, mécaniquement conservés de ce qui est représenté dans notre présent de spectateur. Et dans le même temps, elles ne sont pas leurs sujets. Elles ne sont pas ce qu'elles représentent qui est actuellement absent. C'est pourquoi Bazin considère que la photographie découle davantage du moulage des masques mortuaires que de la peinture. Celle-ci est travaillée par un souci de ressemblance, alors que la photographie serait révélatrice de la réalité du réel, c'est-àdire qu'elle manifesterait le réel en sa finitude : le réel en personne et la personne en réel. Dans une perspective ontologique et psychologique s'inspirant de Mounier et Malraux, Bazin considère que l'absence en jeu dans les photographies et au cinéma, est la mort. Ils ont donc pour fonction anthropologique de nous faire (re)garder ce qui est disparu.

Si Cavell ne conserve pas l'axe ontologique de l'analyse bazinienne, mais s'interroge plutôt sur la dimension analytique et pratique de ces médias ("ce que fait le cinéma", " ce que fait la télévision ») il demeure qu'il considère également qu'un film ne représente pas le monde comme la peinture ou le théâtre. Produite mécaniquement cette image réifie ce qu'elle capte que ce soit un artefact, un paysage, un minéral, un animal, un être humain, mais dans le même temps, elle le personnifie sur le mode de l'absence. Aussi nous montre-t-elle, pour ainsi dire, l'effet de «l'âme » de ce qui a été capté. Néanmoins, plutôt qu'au masque mortuaire, et au problème de la finitude, Cavell apparente cette représentation aux rêves et à notre mise en récit de ceux-ci. Dès lors, cela justifie le fait que l'analyse d'un film ou d'une série ne tende pas à l'exactitude objective, mais autorise tout à fait la production de déformations signifiantes et cohérentes. Lorsque nous nous remémorons un songe et que nous le tronquons ou lui adjoignons des épisodes (par condensation ou déplacement), nous disons quand même quelque chose qui fait sens à partir du rêve déformé lui-même. Même le déni ou le refoulement disent quelque chose du rêve. Et comme pour une bonne part des péripéties de la narration de Lost, nous semble-t-il, ces déformations par reprise des images du rêve ne résultent pas tout à fait d'un calcul délibéré mais ne tiennent pas davantage du hasard.

Cavell précise toutefois que, dans le cas des films, il s'agit plutôt d'un rêve partagé. En d'autres termes, la déformation dépend en sus, de la conversation avec les autres et des normes dégagées par l'échange ; leurs corrections ou approfondissements font sens dans une mise en commun avec les autres spectateurs dont peut faire parti l'auteur ${ }^{13}$. Lost joue beaucoup sur cette dimension d'inconscient commun avec son symbolisme archaïque, ses personnages qui suivent leurs rêves ou qui sont en proie à des hallucinations. Nombre de commentateurs, comme entre autres Briony Addey dans son article «Don't mistake coincidence for fate ", ont souligné l'influence du principe jungien de synchronicité comme thème du récit ${ }^{14}$. C'est aussi un ressort de son écriture et de sa réception, participant à 
son inquiétante étrangeté, à sa dimension ésotérique et initiatique, ressort totalement raccord avec ce que fait le médium télévisuel dont le monde est le purgatoire du réel.

D'autre part comme le souligne Panofsky, le film se distingue du théâtre et de la peinture en ce que l'existence et les différences propres de l'acteur, son corps, ses traits et ses gestes, tiennent une place prépondérante dans sa manière de représenter un rôle et d'être représenté par son rôle. Nous ne reconnaissons pas un acteur de film ou de série, comme nous reconnaissons un portrait peint par sa ressemblance avec un modèle, ni comme nous reconnaissons le rôle d'un interprète au théâtre. Au cinéma ou à la télévision, l'espace est étroitement lié à la durée particulière de prise et de projection (ou de diffusion). De surcroît, le gros plan ouvrant à une proximité inédite avec le visage, le regard et la peau de l'acteur, par la dimension de l'écran, le temps de la prise se fait durée de manifestation d'une pensée subjective (perception, affection et désir). C'est ce que Panofsky nomme «la dynamisation de l'espace » et la «spatialisation du temps». Les plans cinématographiques ou télévisuels rendent ainsi indissociables l'acteur du rôle qu'il joue. Dès lors, l'interprétation cinématographique ou télévisuelle ne s'appréhende pas en fonction d'une relation de modèle réel et de copie artificielle ou manufacturée ressemblante. Elle ne se réduit pas non plus à l'interprétation théâtrale. Dans ce cas, en effet, le personnage existe à l'écrit au préalable et peut être détaché de son interprète, d'autres acteurs ont pu interpréter un même rôle avant lui et l'interpréteront après lui. Pour reconnaître et évaluer l'interprétation, le spectateur a en vue l'identité du rôle dans l'économie de l'intrigue selon le dramaturge, qu'il compare aux variations et particularités, au jeu, que lui offrent la voix, les gestes, la posture de l'acteur et la mise en scène. L'acteur doit être présent quand le spectateur l'identifie dans le temps « continu mais transitoire » de la représentation ${ }^{15}$. La projection cinématographique de l'acteur ou sa diffusion télévisuelle exige la présence du spectateur mais pas celle de l'acteur dont le travail sur le rôle dépend de l'ordre du tournage et de la reprise des rushes par le montage, d'une temporalité discontinue et définitive ${ }^{16}$. Panofsky va donc jusqu'à écrire que le médium audiovisuel est tel qu'il inverse la reconnaissance de la représentation théâtrale : nous reconnaissons l'acteur pour reconnaître le personnage, dès lors l'acteur n'a pas un rôle, il est le rôle et le rôle est lui : « Inutile de dire que ce processus même met en relief la curieuse consubstantialité qui existe entre la personne de l'acteur de cinéma et son rôle ${ }^{17}$ ». C'est ce qui explique, selon lui, que très rapidement les scénaristes ont écrit des rôles pour des acteurs, ce qui, au théâtre, n'était pas impossible auparavant, mais plus rare.

Selon Cavell c'est aussi ce qui explique qu'à l'Âge d'or du cinéma classique américain, et avant durant le muet, on a pu assister à la naissance de «stars »: le public allait moins voir Philip Marlowe, Rick Blaine ou Frank McLoud, qu'Humphrey Bogart voire Bogey, moins la reine Christine ou Ninotchka que Greta Garbo. Hollywood s'attachait donc à développer les possibilités du médium cinématographique, en starifiant, en produisant des individualités, des "types à un individu ", analogues aux anges selon Thomas d'Aquin ${ }^{18}$. Ils étaient des différences formelles projetées sous nos yeux nourrissant un « mythe de la singularité ${ }^{19}$ ", faisant des acteurs, entendus comme noms propres, des modèles d'unicité, des exemples d'êtres originaux. Cela pourrait justifier qu'en voyant Michael Emerson jouer Benjamin Linus, même sans l'avoir vu jouer auparavant dans l'épisode «Sunshine Days» (The X-Files, S09E18, diffusé le 12 février 2002 sur la Fox), dans les épisodes 8 à 13 de la saison 5 de The Practice (diffusés sur $A B C$ de novembre 2000 à janvier 2001) ou Saw (réal. James Wan, 2004) et, après Lost, dans Person of Interest (CBS, 2011-2016), 
nous soyons enclin à le désigner par son patronyme civil tout autant que par celui de son personnage.

Cependant rappelons, si besoin est, que Lost n'est pas un serial de l'Âge d'Or des années 1930 à 1950, même si, dans les années 2000, il a été convenu de parler d'un «nouvel Âge d'Or » avec les séries télévisées. En effet, et toujours selon Cavell, le monde projeté des films hollywoodiens étant éculés, films et séries postérieurs (fin 1950-1970) ont démystifié Hollywood, parfois à leurs corps défendant, en dé-starifiant leurs acteurs. Ceux-ci devinrent des figures indistinctes, des identités sans individualité car toutes égales sous un certain aspect. Méconnaissables, les acteurs apparurent comme des exemplaires interchangeables d'un même standard, d'un même stéréotype disponible. Chacun des acteurs était au mieux une imitation de l'autre sans que nous soyons susceptible de discerner un " original ", c'est-à-dire un prototype voire un archétype : "To impersonate one is to impersonate all; their personalities are already impersonations ${ }^{20}$. " La conséquence fut donc un égalitarisme ou un conformisme disqualifiant des acteurs par leur représentation cinématographique et surtout télévisuelle. Les stéréotypes à l'écran rendant les acteurs eux-mêmes indiscernables les uns des autres présentent une humanité dépersonnalisée, sans différence singulière essentielle sinon numérique, tenant de la foule anonyme où tous se ressemblent.

Cavell va même plus loin. En opposant la starification ou « le mythe de la singularité » des films classiques à l'impersonation des films post-classiques et particulièrement des séries télévisées, il conclut que ces derniers ne sont rien d'autre que l'achèvement de l'égalité entre objets et hommes, qu'instaurent essentiellement les médias photographiques, filmiques, voire pour notre cas électroniques. Si la «starification » d'Hollywood tendait à personnifier, à pourvoir d'une identité singulière, tout ce qui était filmé, et spécialement les acteurs, à l'inverse l'impersonation est associé à un processus de réification, voire de défiguration de l'humain dans lequel, s'accomplirait une dimension totalitaire du monde projetée ${ }^{21}$.

Toutefois et, c'est en cela que films et séries peuvent être considérées comme des opérations esthétiques voire éthiques donnant à penser, certaines œuvres continuent à interroger les potentiels individuant du médium filmique et télévisuel. En effet, elles ménagent une distance critique avec le mythe de la singularité de la "tradition" hollywoodienne, tout en résistant à l'écueil de l'impersonation. Ainsi Cavell, outre des exemples d'acteurs et de réalisateur de films, prend le cas d'une série, pertinent pour la compréhension du problème de l'identité dans Lost - celui de la première saison de Mission Impossible (CBS, 1966-1967). Celle-ci échappe à l'uniformisation de l'image filmique et télévisuelle et donc à l'indifférenciation des acteurs eux-mêmes, mais aussi à la mystification du singulier, et ce de deux manières. D'une part, les sujets du drame y sont les objets, l'action dépendant principalement du bon fonctionnement comme du mode d'agencement des gadgets plus que des motivations et intentions psychologiques clichées des protagonistes. D'autre part, la série s'attache à montrer les identités de ses protagonistes comme se définissant à partir de leur rencontre avec l'autre dans son étrangeté, c'est-à-dire avec autrui : "The fact that the format required the continuing characters to pass as foreigners and, moreover, required one of them to use perfect disguises so that he could temporarily replace a specific foreigner, itself disguised the fact that these characters were already aliens, disguised as humans ${ }^{22}$. "

Mission Impossible développe donc une éthique de l'hospitalité, structurée par l'accueil du guest puisque, dans chaque épisode, la plupart des personnages récurrents de l'équipe 
reçoivent un acteur différent qui fait don de sa singularité à l'épisode. Mieux, l'un des protagonistes, Rollin Hand (Martin Landau), est systématiquement déguisé en " autre ", et reçoit toujours à ce titre en générique l'appellation "Apparition spéciale ", «Special appearance ». Ce procédé met en exergue la tension de la starification et de l'impersonation propre à l'image filmique ou télévisuelle : le spectateur reconnaît Martin Landau en Rollin Hand, le considère comme singulier car justement Rollin Hand est méconnaissable, il n'est pas simplement un "foreigner », un être relativement autre, mais un "alien», une étrangeté. Reconnu de la sorte, il redonne aux autres personnages de l'équipe leur propre singularité. En se manifestant comme rôle, persona, Martin Landau/Rollin Hand renvoie à une personnalité absente, invisible car totalement autre mais déterminant toute identité.

Lost, à la suite d'Alias (2001-2006) de la même famille Abrams et Bad Robot, nous semble prendre acte de la tension starification/impersonation, voire de la « jurisprudence Martin Landau ", en présentant nombre de personnage aux identités multiples et en jouant sur les homonymies, voire le dédoublement d'un des personnages principaux. John Locke et le jumeau de Jacob sont joués par le "même» Terry O'Quinn et tous les autres personnages, en ne s'y trompant pas, s'y trompent quand même puisqu'ils continuent à l'appeler Locke, ce qui fait de lui un « double » dans le sens archaïque du terme ${ }^{23}$.

Dans ce contexte de manifestation de la tension entre starification et impersonation, considérer l'identité télévisuelle à partir de l'homonymie du patronyme d'un acteur jouant un personnage au départ anonyme, puis nommé, mais systématiquement défiguré et refoulé au cours du récit, ne nous parait donc pas incohérent. D'autant que cette homonymie renvoie à un auteur, Ralph Waldo Emerson, pour qui le problème de la représentativité, soit la reconnaissance de sa singularité en régime démocratique égalitaire où sourd le péril du conformisme et de la normalité normative est central ${ }^{24}$. Tel est l'enjeu de ce qu'Emerson nomme le perfectionnisme de la philosophie transcendantaliste prétendant réintroduire de l'héroïsme, du tragique, dans la vie ordinaire. Perfectionnisme qui, selon Cavell dans Les Voix de la Raison, doit beaucoup à la définition que donne Jean-Jacques Rousseau de l'homme comme être naturellement perfectible dans le Discours sur l'origine et les fondements parmi les hommes. Or la série lie les trajectoires du personnage que joue Emerson avec celui d'une femme, Danielle, dont le patronyme (fictif) est Rousseau qui, comme Jean-Jacques, est un être jaloux de sa solitude, légèrement paranoïaque, et qui capturera Benjamin pour le livrer aux rescapés. Quant au lien entre eux, il s'incarne dans un troisième terme qui est le personnage d'Alexandra, la fille perdue de Rousseau, sauvée, élevée et involontairement tuée par Benjamin. Ce personnage médiateur, qui a d'ailleurs le prénom d'un médium (une oracle), puisqu'Alexandra est l'autre prénom de Kassandra, est à la fois cause de la profanation majeure de la série, le meurtre de Jacob, dans l'épisode « The Incident » (S05E17) et de la rédemption de Benjamin Linus/Michael Emerson dans l'épisode « Dr Linus » (S06E07).

La présence ambiguë d'Emerson dans la logique de la fiction est donc liée à la manière dont le médium télévisuel représente la personnalité de ses acteurs : ce que nous voyons à l'écran est plus qu'un simple rôle, cela parle du mystère de la personne humaine en son indéclinable singularité et intimité. Corrélativement, cette présence ambiguë nous incite également, nous téléspectateurs, en reconnaissant cette personnalité, à découvrir la nôtre propre, c'est-à-dire à nous faire confiance, pour entrer dans un dialogue d'âme à âme, de pensée à pensée, à travers un récit se constituant en expérience littéralement télévisuelle. 


\section{Les deux loups}

Chacun s'efforçant de dissimuler comme il peut la finesse de son épiderme et son inaptitude à toute stricte intimité. Mais il n'existe aucun remède qui puisse frapper au cœur de la maladie, en dehors d'une confiance en soi entretenue qui, en pratique, devrait rendre l'individu indépendant de la race

humaine, ou bien une religion de l'amour ${ }^{25}$.

Dans notre article précédent, nous interrogions le traitement audiovisuel par la série du motif kierkegaardien du «saut de la foi » et celui de la «pensée souterraine » rongeant l'œuvre de Dostoïevski, et ce particulièrement dans la saison $2^{26}$. Nous concluions que Lost relie, voire subordonne, le problème ontologique du récit (ce qui nous est raconté existet-il ou non?) et son corrélat "théologique " (enfin la fin tant annoncée de l'Auteur qui raconte et fait autorité ?) et psychologique (y a-t-il quelqu'Un qui reçoit le récit ? Qui suisje moi le récepteur?) à celui éthique, voire métaphysique, de la relation à autrui.

Pour reprendre Pacôme Thiellement, le personnage de Lost est, comme la fiancée du Cantique des cantiques, "malade d'amour ${ }^{27}$ ». Il souffre d'aimer autrui car il ne souffre pas d'être aimé par autrui, et s'invente un passé où cette passion immémoriale est maîtrisée. Il est comme ce sociopathe qui considérait que le moulage de la Méduse qu'il possédait figurait la Mémoire et duquel Emerson déclare : « Il aurait donné son âme pour l'anneau de Gygès. Le malheur qu'il éprouvait d'être visible avait émoussé les affres de la mort ${ }^{28}$ ». La plupart des personnages de Lost sur lesquels un épisode est centré soit par des flashbacks, soit par des flashforwards, puisque cette maladie d'amour est aussi maladie de la mémoire, préfèrent mourir, voire tuer, plutôt que de tolérer l'expérience que voir c'est être $\mathrm{vu}^{29}$. Corrélativement, si la vue est liée au désir, ils ne supportent pas d'être aimés avant que d'aimer eux-mêmes ni de ne pouvoir répondre à l'amour reçu qu'en prenant acte de sa gratuité au-delà ou en deçà d'un retour strictement équivalent, d'un échange réciproque et symétrique. Ils sont aimés autrement qu'ils n'aiment, et n'aimeront jamais en retour pareillement. Pourtant, ils ne comprennent cet écart qu'en y répondant, donc en devant toujours trahir ce don et en en demandant d'avance pardon. D'où cette dimension de repentance constituant le récit en purgatoire et cette identité en rédemption issue du regard que le personnage pose sur lui-même à partir de la vue donnée par autrui ${ }^{30}$. Hormis quelques exceptions, la plupart des personnages ne tolèrent ni d'exister par et pour une vision gratuite d'autrui, ni que la vue leur soit donnée incidemment, en deçà ou au-delà, et malgré un regard autocentré. Ils souffrent donc de ce que leur regard, littéralement, re-garde, et se constitue comme une réponse à ce don de la vue, voire comme une réponse de ce don.

Que le regard soit une réponse au don de la vue, une reprise, explique ainsi également que la maladie de ces personnages soit une maladie de la mémoire, une amnésie autoérotique 31. Ainsi se re-présentent-ils cette gratuité en un incident en se mystifiant, et, selon une logique proche du «Je sais bien mais quand même... » propre au déni, ils s'aveuglent. Ils répètent l'événement en oscillant entre hasard et destin, entre une explication mécaniste et finaliste. Cette oscillation, par voie de réflexion, illustre la réception de la série. Le téléspectateur, après un émerveillement originel accueillant l'interpellation de la promesse, d'un monde de possibles ouvert par l'histoire, ramène cet émerveillement à 
une attente entrant en résonnance avec quelque incident de son propre fonds. Du coup, il se demande si les auteurs savent bien où ils veulent en venir, s'il y a un pilote dans l'avion Lost ou si celui-ci est bien sans queue ni tête ${ }^{32}$. Cette interrogation lui permet certes de justifier vraisemblablement son émerveillement, mais, aussi bien, en en relativisant la nouveauté par comparaison à d'autres récits ou événements déjà connus de lui, le téléspectateur substitue par répétition son Lost partiel et partial : « Nous fabriquons des fables pour cacher la nudité du fait et le conformer, comme on dit, à la loi supérieure de l'esprit ${ }^{33}$ ». La particularité et partialité de la compréhension du téléspectateur se révèle dans une reprise, une relecture et dans la confrontation avec d'autres téléspectateurs, ou avec les rapports d'information qu'il reçoit du contexte d'écriture ou d'intentions avouées des auteurs et acteurs. Mais ces derniers peuvent-ils raconter l'histoire et commenter ce qu'ils racontent dans le même temps, c'est-à-dire sans répéter eux-mêmes d'une manière ou d'une autre ce qui a été reçu?

Les personnages sont tous des incidences: irrésolus comme Jack dans le premier épisode de la seconde saison, ou bien homme de science ou bien homme de foi. Ils se persuadent à la fois que l'incidence était un fait résultant d'une co-incidence : il est l'effet de causes objectives nécessaires statistiquement mesurables, en hommes de sciences. Mais pour autant, ils ne peuvent s'empêcher de croire que l'incidence est une et unique, l'Incident : il leur est destiné ou il est pourvu d'une signification, rétrospectivement attribuée. Ainsi tâchent-ils de stabiliser voire de maîtriser leur passion et s'inventent-ils un moi, une persona souveraine, réifiant corrélativement autrui dans des rapports. Ce faisant, ils récusent toute intimité avec celui-ci en accusant sa singularité, la muant en entité impersonnelle, "the Others", un " on", un «ils ", voire en adversaire omnipotent, un double tragique. Tel pourrait être l'un des sens du Cygne. Dans la pensée New Age dont le Projet Dharma paraît découler, le Cygne est le double cosmique. Le Cygne est le signe brillant brûlant où chaque "enfant du Verseau » peut observer une matérialisation du mythe des âmes sœurs puisque, dans la constellation éponyme, furent découverts en 1971 une étoile et son « double », le premier trou noir ${ }^{34}$.

Le téléspectateur imite le personnage lorsqu'il tâche de maîtriser l'émotion de sa prime réception en interprétant le récit qui, pourtant, par ses chiffres et lieux d'indétermination, le convie à la reprise afin d'échanger avec d'autres téléspectateurs et reconnaître l'autorité des showrunners. C'est ce que nous nommions l'oscillation de la Perle et du Cygne. Le téléspectateur est irrésolu. Ou bien il considère le récit comme un objet, c'est-à-dire comme une juxtaposition de clichés sans rime, ni raison (donc ni auteur), que son désir seul rend signifiant comme la Perle qui, de grains de sables épars, fait sa pleine sphère de nacre. Ou bien il considère le récit comme expression d'un auteur, le chant d'un Cygne qui possède déjà le sens caché de ses paroles et le lui destinait, comprenant, du même coup, le spectateur mieux que lui-même ne se comprend. Dans cette oscillation, téléspectateur et auteur sont des doubles, et Lost, le récit magnétique bipolaire de leur étreinte et lutte tragique, comme Jacob et son double dans le récit, évoquant le combat de Jacob et l'ange du chapitre 32 de la Genèse dont l'issue est l'attribution d'un nouveau patronyme, Israël ${ }^{35}$.

Pourtant l'histoire est porteuse d'un avertissement sur le caractère délétère, schizogène et suicidaire de cette lutte et offre quelque clé à cette inévitable aliénation: le substitut est le candidat, pourrions-nous dire en reprenant les titres des épisodes 4 et 14 de la saison 6. Seul un certain lâcher prise faisant la lumière sur les mensonges dont se berçait le personnage, un renoncement à la maîtrise, le «let go » que nombre de protagonistes 
répètent à Jack, permet de rendre signifiante la souffrance de l'échange du Cygne et de la Perle: «Mais lorsque le fait est examiné à la lumière d'une idée, la fable pâlit et se racornit. Nous contemplons la véritable loi supérieure. Pour le sage, donc, un fait est pure poésie et la plus belle de toutes les fables ${ }^{36}$.» Par ce renoncement, l'échange, la dynamique, des deux stations ne se réduit plus à une lutte pour la reconnaissance ou pour la domination du sens, mais devient bonne réciprocité, et de voyeur nous exhausse en voyant.

De fait, la problématique de la reconnaissance voire de l'intersubjectivité, soi et autrui, soi et les autres, autrui et les autres, est bien au cœur de $\operatorname{Lost}^{37}$. Dans son écriture comme dans la réception qu'elle appelle, la série s'anime d'une tension à la fois démocratiqueégalitaire et aristocratique-distinctive. Comme nous l'écrivions précédemment, notamment avec la référence à Dostoïevski, le récit traite des doubles périls du ressentiment mégalomane et du conformisme pragmatique de nos sociétés démocratiques, et ce afin de penser une relation qui fasse droit au paradoxe de cette grandeur ordinaire que s'efforce de penser Ralph Waldo Emerson. Cette grandeur ordinaire est l'identité où se réconcilient en chacun et entre chacun l'être humain individuel et le membre des nations humaines. Cela correspond à ce que le philosophe nomme dans l'essai Destin, la conduite de la vie, la "suggestion de la double conscience». En reprenant l'image de l'attelage du Phèdre, il compare ce dédoublement au chevauchement alterné de deux chevaux. Ces chevaux sont les parties constituantes de l'âme d'un individu, comme les parties constituantes d'une communauté : le privé et le public, l'individu comme tout et comme partie ${ }^{38}$. Si ce n'est que, pour Platon, il y a une claire hiérarchie entre ces divisions de l'âme et entre les castes de la République - de même qu'une hiérarchie sémantique rigide ordonne la polysémie d'un texte. Le cheval noir et irascible qu'est l' épithumia (la sensualité réactive) est soumis au thumos (courage et bon sens), le cheval blanc et calme qui, lui-même, est soumis au cocher qu'est le noûs (l'intellect). En revanche, contexte démocratique moderne oblige, les deux chevaux d'Emerson paraissent moins distincts, la différenciation hiérarchique plus labile. La tâche du cocher est plus acrobatique, alternative et contextuelle, donc moins systématique et objective. Celui-ci, lâchant prise, louvoie de l'un à l'autre.

Or la narration procède à une telle suggestion puisqu'elle allie une exigence d'accessibilité commune du propos à celle de l'exercice de qualités particulières pour produire une lecture spéciale. L'épisode "The End» (S06E17) se conclut d'ailleurs sur le mariage de la carpe et du lapin, la garde de lî̂le étant laissée à Hugo désignant comme bras droit (« enfant de ma main droite » étant étymologiquement l'un des deux sens du prénom «Benjamin »), Benjamin Linus/Michael Emerson. La question qu'on peut dès lors légitimement se poser est : comment ces deux-là peuvent-ils s'entendre ? Pourquoi eux ? D'un côté nous avons un nerd naïf féru de pop culture (Exposé, Drive Shaft, Back to the Future , Star Wars et manifestement Twin Peaks puisqu'il se fait appeler « Hurley »), quelque peu ignare, interné en raison de crises de mélancolie hallucinatoires, suicidaire car rongé par la peur de blesser autrui. De l'autre, nous avons le politique roué érudit (lecteur de Joyce, Steinbeck, Dostoïevski) méprisant Stephen King (selon les dires de Juliet), celui qui suit "la route antique de l'homme pervers" parricide, homicide, fratricide ${ }^{39}$, infanticide et jacobicide; lui dont l'intrigue de la série accuse la chute jusqu'à l'épisode «Dr Linus » (S06E07). Cette association peut être interprétée comme une mise en abyme du dédoublement auctorial, Cuse/Lindelof, qui a présidé à l'écriture du récit mais aussi à la 
posture dédoublée requise du téléspectateur qui proclamera la "bonne nouvelle» lostéenne, innocent comme un enfant, rusé comme un serpent.

Or, en ne cédant sur aucune des deux exigences opposées, Lost ne se contente pas de produire une double contrainte mais, en l'exhibant, en appelle à un «saut de la foi ${ }^{40}$ ». La référence à la théorie de la double contrainte, se retrouve comme telle dans une autre fiction scénarisée par Lindelof, Tomorowland (réal. Brad Bird, 2015). Rappelons que l'injonction contradictoire: "Soyez vous-mêmes!» est évidemment un exemple de double contrainte souvent pris par Paul Waltzlawick et la psychologie de la communication $^{41}$. C'est également le genre de slogan que Cavell présente comme étant une version dégradée de la « confiance en soi » émersonienne ${ }^{42}$.

Ce « saut de la foi », disions-nous dans notre article précédent, en mobilisant l'influence de Kierkegaard dans la saison 2 (voire dans toute la série), n'est pas tant un renoncement à toute explication rationnelle, ni la promotion d'une logique de l'absurde comme le laisserait croire une certaine acception suicidaire du "Credo quia absurdum» de Tertullien; il s'agit plutôt du « Why not? » de Desmond David Hume, dans l'épisode « Man of Science, man of faith» (S02E01) ${ }^{43}$. Cette réplique n'est pas une fanfaronnade, mais c'est une réponse qui n'en est pas une eu égard à la manière dont Jack, désespéré, posait son problème. C'est-à-dire qu'il s'agit d'une espèce de « Pourquoi pas ne pas ne pas être ? » en réponse au mélancolique "Être ou ne pas être?». Cette réponse est littéralement un encouragement, elle donne du cœur, elle obligeant intimement, de cœur à cœur, à parier que «le pire n'est pas toujours sûr ».

Lindelof dans Tomorowland (réal. Brad Bird, 2015) reformule et radicalise ce processus illustré par la course des deux chevaux d'Emerson par l'analogie du combat des deux loups. Entre un loup désespéré et un loup enthousiaste, qui gagnera ? Celui qu'on nourrit. Ce leitmotiv trouve une variation et un développement sériel dans le problème des jumeaux des épisodes «G'Day Melbourne » (S03E04) et «The Most Powerful Man in the World (and His Identical Twin Brother)» (S03E07) de The Leftovers, explicitant le ressort narratif du roman Bad Twin de "Gary Troup ». Ces paradoxes en appellent à une éminente responsabilité métaphysique de l'optimisme, à une bonté intérieure, plus qu'à une nécessité simplement logique et conséquente, ou à un impératif moral formel de faire le bien. L'optimisme ici invoqué est l'encouragement, il s'apparente à une "confiance en soi » (self reliance) créatrice équivalent à une dynamique de résilience, ce qu'Emerson nomme le perfectionnisme indissociable d'une confiance en autrui. Celle-ci, par un acte probable, mais qui nous semblait a priori théoriquement impossible, voire moralement irresponsable, nous permet d'envisager une situation perdant-perdant autrement, la transfigurer en la rendant gagnant-gagnant et signifiante, comme dans l'ironie ou l'humour avec le nonsense, voire l'illumination poétique et philosophique ${ }^{44}$.

41 Toutefois cela implique aussi la possibilité de perdre sur les deux tableaux, c'est-à-dire de susciter le ressentiment de celui qui confond la confiance en soi et le complexe de supériorité, l'amour propre. La confiance en soi n'équivaut pas simplement à clamer haut et fort que l'on est "spécial». Il y a aussi un conformisme de l'anticonformisme également aliénant et suicidaire qui convoque la présence de l'autre pour la répudier, et se nier par là même. Cette attitude paraît d'ailleurs sanctionnée par la série, étant principalement le fait de deux personnages : Locke et le jumeau de Jacob (Titus Welliver) qui devient le double de Locke à partir de la fin saison $5^{45}$. Il est significatif que le seul moment où l'énonciation du terme «special» n'est pas associée à un comportement mégalomaniaque ambivalent sorte de la bouche d'un Benjamin repentant pour 
reconnaître la singularité de Locke en s'excusant dans le flashsideways de l'épisode «The End» (S06E18), avant de dire à Hugo sur l'île qu'il y a sûrement une autre manière de la garder que celle de Jacob et de rajouter "A better way."

Lost comporte ainsi des éléments de récit stéréotypés et, dans le même temps, ne se contente pas des " grosses ficelles » du sens commun à base de twists aussi spectaculaires que répétitifs au service d'un pathos très accessible et efficace pour drainer un large public. En parsemant les épisodes de chiffres, de références érudites et occultes, en jouant la carte des lieux d'indétermination elliptiques et allusifs, les auteurs nous invitent à une archéologie desdits stéréotypes et à revoir nos « classiques ». Le climax est donc ici tout autant un effet narratif nous captivant qu'il est, étymologiquement, un « degré » vers un autre niveau de lecture nous appelant à nous délivrer de cette prime captation d'attention. Il incite à recevoir le récit de façon plus active voire plus aristocratique, resserrant le cercle de téléspectateurs toujours plus spécialisés, des losties. Ce redoublement d'attention exigé, cette explication, permet de pénétrer et d'ordonnancer la polysémie du propos afin d'adopter un regard sur le médium: de voir ce que fait la série, ce que fait la télévision, ce que font culture populaire, savante et traditionnelle. D'autres questions apparaissent comme appels de nouveaux horizons. Cela stimule l'intelligence réceptrice qui analyse et synthétise séquences et épisodes en fonction d'indices (des récurrences, des ellipses, des événements qui ne " collent " pas, suscitant correspondances et analogies) et qui s'élève, à coup d'hypothèses et théories dans des dimensions de sens toujours plus larges. Pour les produire, le téléspectateur lutte avec lui-même, comme Jacob et son jumeau, et s'arrache de sa compréhension première pour s'exhausser à un niveau de sens plus compréhensif encore, regardant, rétrospectivement, l'analyse précédente comme une Station. Pour cela il s'aide intuitivement, ou très explicitement, des quatre sens que dégage Dante dans Le Banquet (littéral, analogique, moral et eschatologique) et qu'expose Pacôme Thiellement dans l'étude qu'il produit sur Twin Peaks, série dont l'influence sur Lost ou The Leftovers est incontestable de l'aveu même de Lindelof ${ }^{46}$.

Ce faisant, la contrepartie de la découverte de cette hiérarchie sémantique est que le regard du téléspectateur tend à oublier son effort d'élucidation antécédent et en vient à considérer comme accessoires les niveaux de sens les plus évidents et visibles, ceux qui sont au bas des six degrés de l'échelle de Jacob. Or, dans la littérature mystique, l'ascension spirituelle du Cantique des cantiques, ou de l'échelle de Jacob du chapitre 28 du Livre de la Genèse est souvent opposée à celle de la Tour de Babel du chapitre 11 du même livre : le risque serait de voir le " $7^{\text {ème }}$ ciel » de la série comme une ultime Station où tout sera élucidé où, enfin, le téléspectateur «se fera un Nom » et sera l'égal de l'Auteur avec un grand « $\mathrm{A}$ » qui a pouvoir et liberté, qui est voyant invisible. Las! Dans l'épisode « The Incident » (S05E16), Jacob meurt assassiné par ceux-là même qui croyaient le plus en lui et ont tout perdu, déclarent-ils, en son nom ; sixième saison, sixième barreau, accrochezvous au pinceau, aux branches, à tout ce que vous pouvez, Cuse et Lindelof enlèvent l'échelle! Plus le téléspectateur s'est élevé dans la hiérarchie des niveaux de lecture, plus dure est la chute, plus il pensait s'être distingué et avoir obtenu de la série une confirmation qu'il était "spécial », une reconnaissance de son intelligence, une autorité relative, plus âpre parait être le ressentiment.

En effet, la saison 6 poursuit la profanation amorcée à la fin de la saison 5 et navigue apparemment à vue. Avec ses flashsideways, elle relance éhontément l'hypothèse de l'île purgatoire, prétendument démentie à maintes reprises par les showrunners comme 
ironiserons les auteurs des Simpsons dans l'épisode « Beware my cheating Bart » (S23E18) diffusé sur la Fox le 15 avril 2012 $2^{47}$. Autour d'un feu de camp à la Survivor, dans l'épisode "What They Died For» (S06E16), les rescapés sont finalement définis par Jacob comme des « candidats » destinés à la garde de l'île car sinon leur vie n'aurait pas eu de sens. On s'attendrait presqu'à ce qu'un numéro de téléphone apparaisse au bas de l'écran pour que le public choisisse qui évacuer après le speech du présentateur. Par voie de réflexion, les auteurs faisant manifestement du téléspectateur le dépositaire du récit, beaucoup ont vu dans Lost l'histoire d'une démission voire d'un mépris et, dans son jeu de piste, un loophole voire long con digne de Sawyer (Josh Holloway), un étalage d'érudition déployé au mieux avec désinvolture, au pire comme une imposture ${ }^{48}$. Le récit n'aurait été qu'écran de fumée, puisque se ramenant au récit stéréotypé pré-senti dès la première saison, tous ses secrets archaïques de fantasy et ses « règles » correspondant quasiment à celle d'un reality show des plus ordinaires. D'autre part, un reality show prétend faire de la télévision un médium bottom up, censément interactif, démocratique, type jury populaire; son « intrigue » suppose la participation directe des téléspectateurs pour éliminer ou garder son candidat, tandis qu'ici le récit suppose une verticalité qui ne paraît pas s'assumer puisqu'elle se présente comme un cadeau.

Recevoir un don a quelque chose d'humiliant écrit d'ailleurs Emerson. Cela implique que le donataire suppose en quelque sorte, sinon une supériorité physique ou morale du généreux donateur, au moins une asymétrie telle que ce dernier peut passer pour quasiment indépendant, "special». Cette terrible libéralité oblige le récepteur à une reconnaissance plus profonde que dans un échange économique, d'où une certaine justification du sentiment d'ingratitude ${ }^{49}$. En effet, puis-je répondre à quelqu'un qui répond du fait que je réponds de moi ? Comment me montrer reconnaissant sans mesurer le bienfait et rabaisser ce don à ce que nous nommions un rapport et donc le profaner? Du coup, il est légitime de se demander si ce temps « perdu » ne servait à rien puisqu'au final le spectateur apparemment le plus anonyme, le plus littéral et «inculte » à l'instar de Hugo peut jouir du récit autant que le lostie le plus spéculatif et méritant, le plus au fait de ses chiffres, comme Benjamin. Rajouter à cela que tout ce temps dépensé à voir était un don et que nous sommes alors appelés à garder la fiction, c'est entériner de façon gratuite, gracieuse, démesurée, l'autorité de showrunners et donc disqualifier le téléspectateur. On nous répondra qu'Hugo peut garder l'île car il aime l'histoire et ses personnages, écoute chacun sans vouloir imposer sa lecture, il est humble de cœur, ce qui n'est manifestement pas le cas de Ben jusqu'à la saison 6, ni même de la plupart des homonymes de personnalités historiques qui jalonnent le récit. Par voie de réflexion, Lost nous invite à relativiser la valeur de la culture savante : "Ce que pense la pensée n'a aucune importance à côté de la vérité que nous sommes et qui s'affirme en silence ; si, après cinquante ans de solitude, Epictète, Goethe et saint Paul abordaient en mon île, ils ne pourraient me dire que ce que me dirait en même temps et plus immédiatement peutêtre le petit mousse de leur navire ${ }^{50} »$.

Sans doute une lecture trop logique ou géométrique encourt-elle le risque d'une acception instrumentale de Lost, comme machine à produire du divertissement, que celuici soit prosaïque ou raffiné. Cette lecture cherche une clé des secrets pour en finir avec Lost, pour s'empêcher de se demander si le secret n'est pas dans le fait de la vanité d'une telle attitude avide d'explication toute faite et définitive. Cela ne passera pas, et l'amertume contre la série est, comme le refoulement, encore une manière de la laisser travailler en nous... Aucune interprétation n'exempte totalement de l'expérience, de voir 
et revoir la série, de la souffrir comme trahison des personnages avec les personnages, des auteurs avec les auteurs, des téléspectateurs avec les téléspectateurs, commémorer, re-garder. Il faut s'avouer et répondre de cette douleur. Le récit implique donc une part érotique, de philia voire d'agapè, c'est-à-dire de poésie, de sentiment et d'imagination, un logos qui ne se constitue pas en rupture totale avec le muthos, laissant une place au cœur, à la passivité voire à la douleur. Cela nous semble être attesté par le fait que la plupart des livres mentionnés à l'écran, ou par les intitulés des épisodes, sont des œuvres de fiction de la pop culture du genre science-fiction, fantastique, quelques "classiques" romanesques et théâtraux, et la Bible. Le seul ouvrage labellisé " philosophique » est Crainte et Tremblement de Kierkegaard, trouvé dans le sac de Montand non loin de son bras gauche sous le mur du Temple, et encore, en français dans le texte, sachant qu'il s'agit d'une écriture assez ironique ${ }^{51}$.

La narration ne semble pas tendre avec une culture savante exclusivement théorique et utilitaire. Si l'homonymie des noms de certains personnages avec des scientifiques ou des philosophes peut nous amener à penser que l'île est comme la République des Lettres, une société d'hommes d'exception, le monde de demain, force est de constater que la plupart d'entre eux finissent par mourir, souvent bêtement et sans bien comprendre ce qui leur arrive. Lost n'est cependant pas absurde, et ce traitement n'est pas pour autant un éloge de la bêtise conséquence d'un ressentiment, d'une résignation conformiste. Certes, tout se passe comme si les auteurs proposaient à un public érudit des points d'entrée pour voir autrement la série, en adoptant le regard de "grands hommes", et les sacrifiait systématiquement montrant leur inaptitude à saisir le monde du récit pour donner la garde de l'île à un perdant. Néanmoins, ce n'est pas une négation de l'aspiration à la grandeur, ce n'est pas parce qu'il n'y a pas de cité idéale ou d'utopie.

Ces références ne sont pas « grandes » dans le sens où l'entendrait une histoire officielle. Une histoire officielle ou édifiante, entendue comme récit clos, justifie généralement l'anticonformisme passé de la personnalité et son effort pour être reconnu et devenir soi, en un conformisme rétrospectif. Elle fait alors de cette personnalité, un " grand homme ", un classique, un monument. Du coup, elle tend à n'oublier que mieux la douleur vécue de la gestation de cette personnalité. L'homonymie dans la fiction nous rappellerait ainsi que l'identité des « personnalités » n'a pas toujours été acquise, que l'historiographie dépend d'une pratique de la mise en récit et donc d'un rapport affectif à celui-ci, que, comme nous le soulignions auparavant, le regard dépend d'une patiente réception du don de la vue.

L'originalité de l'écriture d'Emerson, selon Cavell, est en cela éclairante sur l'expérience du rejet. Emerson aurait écrit et se serait donné lui-même à lire parce qu'il souffrait d'être rejeté, en but à un conformisme niant son unicité. Toutefois, il ne voulait pas représenter un stéréotype d'anticonformisme et asservir son lecteur à ce nouveau conformisme. Aussi ne se justifiait-il pas dans un langage ésotérique au sens parfaitement maîtrisé où se seraient affirmés l'autosuffisance d'un moi souverain et donc son rejet des autres, en mesure de rétorsion au rejet qu'il ressentait. Bien au contraire, même s'il convoquait des références extrêmement érudites, à l'instar de Montaigne, il s'exprimait dans un langage ordinaire, presque anodin, au risque de l'insignifiance, au risque d'être rejeté par un lecteur qui voudrait par trop se conformer à lui. Par ce procédé, il inciterait son lecteur à la confiance en soi, c'est-à-dire à répondre intimement de ce qu'il lit, à chercher lui-même un autre sens, en prenant éventuellement la plume ou la parole à son tour. L'écriture de Lost nous parait inspirée par ce procédé. De la sorte, nous, auteurs, 
acteurs et téléspectateurs, partageons les émotions proposées dans le récit, et donnons un sens à la souffrance d'où s'initiait la narration : « La douleur est fonction de l'idée qu'il n'y a aucune raison pour nous de ne pas entrer dans le monde à venir, aucune raison qu'il ne soit pas réel, et donc que je suis forcément en train de le rejeter, et de rejeter l'existence des autres en lui ; et que les autres sont en train de rejeter mon existence en ce mondelà $»^{52}$, nous dit Cavell.

Dans cette optique, on peut comprendre pourquoi Benjamin Linus/Michael Emerson est le souffre-douleur de la série, « Benjamin » signifiant aussi étymologiquement « enfant de ma douleur ", sa position malaisée d'antagoniste sa chute et sa rédemption, signifient cette épreuve du rejet (rejeter et être rejeté) nécessaire à la découverte de son identité profonde, comme l'est d'ailleurs, selon Cavell, l'autorité d'Emerson dans l'histoire de la philosophie et par rapport à notre propre compréhension de nous-mêmes via son œuvre.

\section{Une lumière aveuglante}

L'homme sage reste chez lui, dit le vieux professeur. C'est d'Emerson, il y a beaucoup de vrai dans cette pensée.

Pas si l'on en croit votre héros, Ulysse.

Oh ! Il a fini par en voir la sagesse lui aussi. ${ }^{53}$ Les heures héroïques sont moins apparentes, celles de l'abnégation ne sont pas encore revenues; il ne nous reste plus que la vie quotidienne, et cependant nous ne pouvons vivre sans grandeur. Il [Emerson] a donné un sens presque acceptable à cette vie qui n'avait plus ses horizons traditionnels, et peut-être a-t-il pu nous montrer qu'elle est assez étrange, assez profonde et assez grande pour n'avoir besoin d'autre but qu'ellemême ${ }^{54}$.

Dans l'une de ses premières œuvres théâtrales, Les Aveugles (1890), Maeterlinck se propose de revisiter La Tempête de Shakespeare, à l'aune du chapitre 15 de l'Evangile de Matthieu et de la philosophie d'Emerson. Sur une île, alors que mugit une tempête qui approche, douze aveugles sont perdus : six hommes, six femmes et un bébé dont s'occupent l'une d'elle que tous les autres traitent de folle. Ils racontent qu'aucun d'eux ne se souvient de la manière dont ils sont arrivés là et, assis, attendent leur guide, le prêtre en charge de l'hospice, que le spectateur aperçoit au milieu d'eux, immobile car mort. Cette intrigue, faisant manifestement du surplace, amènera ces « héros " à découvrir ce que n'importe qui voit dès l'ouverture du rideau, et ce grâce à l'intervention d'un chien. Qu'adviendront-ils à la fin ? On entend des bruits se rapprochant, vient-on les sauver ou n'est-ce que le vent? Les aveugles brandissent le nouveau né voyant et s'affrontent à l'inconnu... Ce drame sur la présence de la mort indissociablement liée à une incapacité fondamentale à communiquer et reconnaître autrui, parait faire un deuil, relatif, du merveilleux de La Tempête et, sous l'impulsion d'Emerson, ramener les vestiges de l'imaginaire encore traditionnel et occulte de Shakespeare à une portion congrue. Cependant en l'ancrant dans une scène quasi-quotidienne, il renoue avec le dramaturge anglais en renouvelant le mystère de sa romance $e^{55}$. 

Emerson ne détruit pas tant tout imaginaire ni toute autorité, qu'il annonce une autre manière, une autre voie de la " grandeur ", une autre manière de l'exercer au quotidien et d'être reconnu. C'est ce qu'il dit à Hugo à propos d'une meilleure manière de faire que celle, tragique, de Jacob, « a better way ». Une autre voie suggérée dans l'épilogue The new man in charge dont Benjamin Linus/Michael Emerson est le principal acteur et qui serait une réconciliation poétique avec un monde pourtant démystifié. Subséquemment nous comprenons pourquoi la saison 6 s'apparente à une profanation: les flashsideways nous ramènent au quotidien le plus prosaïque des « rescapés » qui ne le sont pas, puisque l'île a été détruite comme l'atteste le travelling sous-marin de l'épisode «LAX » (S06E01), tandis que la symbolique archaïque et traditionnelle sur l'île périclite. Dans cette dernière saison, le dédoublement de conscience et l'indétermination quant au réel et à l'imaginaire parait à son comble : s'agit-il d'un récit de fantasy archaïque où des héros rêvent qu'ils ont échappé à leur destin pour y consentir au final? S'agit-il d'un récit de science-fiction nous montrant deux trajectoires réelles divergentes à partir d'un même moment du temps faisant droit à la liberté des protagonistes ? ${ }^{56}$

Sans doute là encore s'agit-il d'un rêve partagé où l'opposition ordinaire/extraordinaire nous permet de mieux comprendre l'opposition imaginaire/réel. Ainsi est-il confirmé que Lost comme expérience télé-visuelle est ironiquement un reality show, le spectacle de la réalité est la réalité du spectacle. Les flahsideways nous permettent de critiquer l'univers traditionnel et mythologique de l'île de le rendre plus accessible comme un reality show, mais, réciproquement, cet univers merveilleux dans son essoufflement, sa vulnérabilité, son appel à la défense instille de l'héroïsme et de l'ésotérisme dans des situations communes. Il inocule de l'héroïsme chez un médecin divorcé qui trouve le courage de parler à son fils en comprenant qu'il intimidait son père défunt, chez une fugitive qui va aider une femme enceinte et se prouver qu'elle est responsable, chez un policier orphelin traumatisé comprenant, en voyant La Petite maison dans la prairie, que son donjuanisme, est mépris d'avance, rejet par peur d'être lui-même rejeté, chez un professeur d'abandonner ses ambitions pour aider une élève... Dans chacune de ces situations, les personnages s'efforcent de répondre du fait qu'ils sont vus par autrui avant de le voir et de se voir eux-mêmes. Ils s'efforcent de répondre à, et de répondre de, la dissymétrie d'une relation avec autrui. Or ces situations sont mises en correspondance avec le «même» problème sur l'île, les personnages doivent répondre du fait qu'ils sont destinataires d'une Lumière, du regard invisible du tout Autre qui les voit avant qu'ils ne se voient, mais ce avec un accent extraordinaire porté sur la dissymétrie. En répondant de cette relation de l'ordinaire (comme relation avec autrui), et de l'extraordinaire (comme relation avec l'Autre), de cette relation des "petits» et des "grands» sacrifices, les personnages, les téléspectateurs et les auteurs de retour au quotidien, peuvent accéder à une dimension de conscience supérieure, à l'éveil de son Soi, devenir voyants. Nous, auteurs (équipe de tournage comprise), acteurs, téléspectateurs, sommes amenés par le récit même à nous demander : pour qui est-ce que les auteurs se donnent à voir et donnent à voir les téléspectateurs en envisageant les personnages du récit? Pour qui est-ce que les téléspectateurs se donnent à voir et donnent à voir les auteurs en envisageant les personnages du récit? Cette question nous encourage à contempler la réalité du réel derrière la représentation imaginaire égocentrique et conformiste que l'on s'en faisait. Elle nous encourage à nous souvenir avec gratitude de cette relation de l'ordre du don, en nous ouvrant la perspective d'un pardon de notre ingratitude par notre reprise du récit. 
54 En définitive, quel rapport y a-t-il entre le problème de l'individuation par l'image cinématographique ou télévisuelle et la confiance en soi (self reliance) ? Là encore Lost offre un terrain de réflexion fécond puisque le parcours de Benjamin vers la reconnaissance, sa chute et sa rédemption jusqu'à sa coresponsabilité de l'île, incarne les deux faces de l'interprétation de la philosophie d'Emerson: celle, réductrice, d'un individualisme politique et moral reposant sur la certitude de l'existence du «moi » et celle plus métaphysique d'une spontanéité fondamentale tenant d'un «Soi » non immédiatement conscient, en intimité avec une destinée naturelle et autrui. On a une opposition proche de celle qu'établit Nietzsche (grand lecteur d'Emerson à l'instar de Heidegger) entre la « volonté de pouvoir » et « la volonté de puissance ». Si ce n'est que la version du destin que propose Nietzsche, l'Éternel Retour du même, est essentiellement tragique archaïque à l'instar de son Surhumain, tandis que celle que propose Emerson est tragique ordinaire; la confiance en soi du "grand homme " paraît indissociable de la sympathie, et de ce qu'Emerson nomme « une religion de l'amour ».

Néanmoins cette comparaison à Nietzsche et cette ambivalence de la confiance en soi métaphysique entre "grand homme ordinaire » et "surhumain ", sont sans doute l'un des aspects du refoulé de la série les plus significatifs. Il est en effet remarquable que dans un récit qui brasse autant de références classiques et philosophiques internationales, il n'y ait à aucun moment (à ma connaissance) de référence patronymique au moindre penseur allemand - si ce n'est éventuellement, Benjamin qui, de prénom dans la série, renverrait au patronyme historique de Walter Benjamin. Le philosophe de l'École de Francfort était spécialisé dans l'esthétique, considérant le cinéma tout autant comme un instrument d'asservissement des masses, celui de la perte de l'aura de l'œuvre, qu'un médium permettant de redécouvrir le potentiel interactif de la narration orale, ouvrant à l'aura d'une nouvelle communauté ${ }^{57}$. Traqué par les nazis, car juif, et arrêté par la police espagnole franquiste, Benjamin se donna la mort en 1940, plutôt que de tomber entre les mains de la Gestapo. En s'en tenant au patronyme néanmoins, ce silence quant à la culture germanique est assez éloquent quand on sait que la série véhicule à plusieurs reprises, les relents d'une imagerie voire d'un imaginaire totalitaire et concentrationnaire, comme le soulignait Ophir Lévy dans une conférence intitulée «Le Projet Dharma: la boucle étouffante du Même » en juin 2014 à l'université de ParisSorbonne. Dans l'épisode «The Man behind the curtain ( (S03E20) centré sur Benjamin Linus/Michael Emerson, ce dernier dévoile à Locke une fosse commune dans laquelle s'empilent, à ciel ouvert, les cadavres des membres du Projet Dharma préalablement gazés. Nietzsche citant Emerson dans un essai intitulé Fatum, Histoire et Pensée assimile la volonté de puissance à la confiance en soi comme destruction du passé et de l'histoire, cette « auto-aliénation onirique », comme tombée de rideau vers la création d'un nouveau monde.

Cette chute du «moi » avide de pouvoir, est paradoxalement ce qui permettra à Linus/ Emerson de passer d'une face à l'autre pour se relier à son Soi. De la saison 2 jusqu'à la fin de la saison 5, Benjamin est un personnage qui s'efforce de manipuler et de calculer le comportement de son entourage au profit revendiqué de Jacob, dont il prétend appliquer les règles, le droit. Mais plus il pense dominer la situation, être un self made man, en tuant son père et sa «famille » ou en s'autoproclamant père lui-même en volant Alexandra à sa mère, plus il se rend tributaire de l'image de pouvoir qu'il s'est donné et s'oublie profondément. D'où la question que Jacob lui pose lorsque, couteau à la main, il lui 
demande des comptes ; le « What about you? », qui répond au " What about me? », interpelle Benjamin en son identité profonde, en sa personnalité.

Or, cela rejoint ce que nous affirmions sur la tension de l'image cinématographique ou télévisuelle entre la starification et l'impersonation quant à la reconnaissance de l'identité des sujets filmés et qui est une manière de montrer le paradoxe de l'individualisme démocratique et concurrentiel où tout le monde est unique car tout le monde se conforme pareillement à un souci de se distinguer. L'identité de l'homme télévisuel, de l'homme en régime démocratique n'est pas acquise: plus elle aspire au retour au moi starifié de l'Âge d'or, plus elle est en but à l'impersonation. Les mesures esthétiques nous amenant à nous rendre compte de cette tension, comme la «jurisprudence Martin Landau ", les homonymies heureuses ou les incidents de frontière entre fiction et réel nous paraissent invoquer un Soi qui suppose une relation réciproque avec Autrui, une intimité.

L'Âme ou le Soi de Ben, son ange gardien, c'est Alex qu'il sacrifie pour l'île et qui se sacrifie pour lui. Alex est la seule qui le défend quand tout le monde veut le tuer dans l'épisode « Confirmed Dead» (S04E02) et c'est en son nom qu'il ne tue pas Ilana (Zuleikha Robinson), l'«Alex » de Jacob, dans l'épisode « Dr Linus » (S06E07). La mort donnée de l'adolescente réconcilie les exigences du respect du nom du père et du mariage, évoquées en première partie ; c'est, en effet, la fille qui fait de Benjamin son père et qui, dans les flashsideways lui ouvre la perspective d'une union avec Rousseau. Outre qu'Alexander est le prénom du bon «mauvais » jumeau Widmore, le sinistre gaucher sur la voie de la rédemption dans Bad Twin, le prénom Alexandra est aussi celui de la princesse Kassandra. Dans la mythologie grecque et les tragédies, cette dernière voit toujours ce qui va se produire, généralement le pire, et cela advient car elle n'est jamais crue par son entourage qui ne change pas son comportement. En cela elle est une figure tragique, ce qu'elle a prédit a toujours été vrai car elle échoue à modifier le cours des choses. Or, dans la série, Alexandra met en garde son père de la même manière en lui disant que, par sa soif de contrôle, il est le véritable danger pour l'île. Pourtant elle n'est pas écoutée car ce dernier veut absolument la protéger et, "innocent coupable ", de ce fait même, il la tue dans l'épisode bien nommé « The Shape of things to come » (S04E09).

L'intitulé de cet épisode fait référence à un roman d'anticipation de H.G. Wells (lui-même inspiré par Le Temps et le Rêve de J.W. Dunne) où le narrateur lit le journal d'un ami qui, en rêve, aurait consulté un recueil d'histoire composée d'images animées se commentant elles-mêmes, des images qui sont du "future past». Cette référence nous explicite également le régime temporel bifide de l'image télévisuelle : ce futur antérieur désabusé, ce fatalisme quasi-onirique est caractéristique du dédoublement du récit en présent insulaire, d'une part, et flashback ou flashforward, d'autre part ; dédoublement par lequel les personnages répètent un incident pour le maîtriser et ne forgent leur identité que sur le malheur à venir qu'ils s'étaient promis.

Mais, ce faisant, en exhibant ce fatalisme et notre logique tragique de prédiction autoréalisatrice, la série nous ouvre à la perspective de la confiance en soi pour être voyant. Dans le dernier essai du Trésor des humbles, La Beauté intérieure, Maeterlinck compare notre Âme, notre Soi ou identité profonde à Kassandra en exil, persécutée, sous le porche des Atrides qui anticipe ce qui va se produire. Elle prophétise par signes dans un langage poétique voire onirique, celui de la beauté et de l'amour. Ces signes, le moi, comme Agamemnon ou Priam, ne les reconnaît pas car trop logique et autoritariste, il est effrayé par le lâcher prise ${ }^{58}$. Mais même refoulée Kassandra demeure et son travail est comparé à 
celui d'une perle qui, des blessures que le moi s'inflige, tâche de produire une beauté plus profonde rendant possible une rédemption: "Est-ce que le mal même ne devient pas précieux, lorsqu'elle (l'Âme) en a extrait le diamant profond du repentii ${ }^{59}$ ? » Maeterlinck cite alors Emerson expliquant que les évènements de notre vie qui nous apparaissent comme "forme inerte" prendront aussi «leur essor " pour signifier notre existence positivement. Ainsi de "form ", ces épisodes constituant mon moi deviendront-ils «shape " c'est-à-dire ils signifieront en faveur de la vie du Soi pour peu que le moi lâche prise et se recueille sur sa patience, le fait d'être vu pour voir. Si Kassandra est une voyante, c'està-dire qu'elle anticipe sur ce qui sera pour le faire être, alors ce n'est que par un "saut " qui rejette notre rejet, ce n'est que par l'anticipation de quelque chose d'improbable, que nous nous mettons en demeure de faire advenir rétrospectivement, de recueillir, une signification dans une situation qui nous semblait sans issue. Concluons avec Cavell citant Emerson:

Pour moi, le rapport avec Emerson est que ce monde meilleur «que nous pensons ", et dont nous savons qu'il n'existe pas, alors qu'il n'y a pas de raison acceptable qu'il n'existe pas, n'est pas un monde disparu et dont il faudrait faire son deuil, mais un monde à porter dont il faut témoigner ${ }^{60}$.

\section{BIBLIOGRAPHY}

BAZIN André, Qu'est-ce que le cinéma ?, Paris, Cerf, 1999 [1975].

BÉGHIN Cyril, « Faire une série », Cahiers du Cinéma, n735, Juillet/Août 2017, p. 14-15.

BENJAMIN Walter, Euvres III, Paris, Folio, 2001.

BLANCHOT Maurice, L'espace littéraire, Paris, Folio, 2014 [1988].

CAVELL Stanley, The World Viewed, Enlarged Edition, Harvard, Harvard University Press, 1979 [1971].

__-_. Statuts d'Emerson : Constitution, Philosophie, Politique, Combas, L'Eclat, 1992.

_-_. Conditions nobles et ignobles : la constitution du perfectionnisme moral d'Emerson, Combas, L'Eclat, 1993.

--_. Les Voix de la Raison: Wittgenstein, le scepticisme, la moralité et la tragédie, Paris, Seuil, 2012.

CORCUFF Philippe et Sandra LAUGIER, « Perfectionnisme démocratique et cinéma : pistes exploratoires ", Raisons politiques, vol. 38, $\mathrm{n}^{\circ} 2,2010$, p. 31-48.

CORNILLON Claire, « Lost, fiction kaléidoscopique », TV/Series, hors-séries n¹, 2016, http:// tvseries.revues.org/1632.

DERRIDA Jacques, Donner la mort, Paris, Galilée, 1999.

DOSTOÏEVSKI Fiodor, Les Frères Karamazov, Paris, Folio, 1994 [1973].

DULONG Guillaume, « La promesse de Lost (À propos de la Saison 2) », TV/Series, hors-séries n¹, 2016, http://tvseries.revues.org/1636. 
EMERSON Ralph Waldo, Société et Solitude, Barcelone, Payot-Rivages, 2015.

_-_. La Confiance en soi, Barcelone, Payot-Rvages, 2000.

FAVARD Florent, « “You can('t) let go now”: Mystery boxes, quantum readings and very bright lights in Lost »,TV/Series, hors-séries $n^{\circ} 1,2016$, http://tvseries.revues.org/1738.

GREEN Eugène, Présences : Essai sur la Nature du cinéma, Paris, Desclée de Brouwer/Cahiers du Cinéma, 2003.

HATCHUEL Sarah, Lost : Fiction vitale, Paris, PUF, 2013.

HATCHUEL Sarah et Randy LAIST, « Lost : une « romance » shakespearienne ? », TV/Series, horsséries $\mathrm{n}^{\circ} 1,2016$, http://tvseries.revues.org/1656.

HUME David, Système sceptique et autres systèmes, Paris, Seuil, 2002.

KAYE Sharon, éd., Lost and Philosophy: Think together, Die alone, Hoboken, John Wiley and Sons, 2010.

KIERKEGAARD Søren, Crainte et Tremblement, Paris, Payot-Rivages, 1999.

LAUGIER Sandra, «Emerson, père fondateur? ", Raisons politiques, n²4, novembre 2006, p. 9-31.

MAETERLINCK Maurice, Petite Trilogie de la mort : L'Intruse, Les Aveugles, Les Sept Princesses, Bruxelles, Espace Nord, 2012.

_-_. Le Trésor des Humbles, Paris, Grasset, 2015 [2008].

MOURLET Michel, L'Écran éblouissant : Voyages en cinéphilie 1958-2010, Paris, PUF, 2010.

NOGUEZ Dominique, Cinéma, Théorie, Lectures, Paris, Klincksieck, 1973.

PLATON, Phèdre, Paris, Garnier-Flammarion, 1992.

SCHIFF Jean-Marie, L’Âge Cosmique aux USA, Paris, Albin Michel, 1981.

THIELLEMENT Pacôme, Les Mêmes yeux que Lost, Paris, Leo Scheer, 2014.

_-_. La Main gauche de David Lynch, Paris, PUF, 2016.

_-_. " Lost ou le Vade-mecum des malades d'amour », TV/Series, hors-séries n¹, 2016, http:// tvseries.revues.org/1614.

TROUP Gary, Bad Twin, Paris, Fleuve Noir, 2007.

WATZLAWICK Paul, Faîtes vous-mêmes votre malheur, Paris, Seuil, 2014.

\section{NOTES}

1. Ralph Waldo Emerson, «Expérience » compris en supplément dans le recueil de Stanley Cavell, Statuts d'Emerson : Constitution, Philosophie, Politique, Combas, L'Éclat, 1992, p. 71.

2. Jeremy Barris, «Lost and the question of life after birth", in The Ultimate Lost and Philosophy: Think Together, Die Alone, éd. Shanon Kaye, Hoboken, John Wiley \& Sons, 2011, p. 107-119.

3. Ralph Waldo Emerson, La Confiance en soi et autres essais, Barcelone, Payot-Rivages, 2015, p. 85.

4. Ibid. p. 85-127.

5. Maurice Blanchot, L'Espace littéraire, Paris, Folio, 2014, p. 223. Guillaume Dulong, « La promesse de Lost (À propos de la Saison 2) », TV/Series [En ligne], Hors séries 1 | 2016, mis en ligne le 14 Octobre 2016. URL : http://tvseries.revues.org/1636.

6. Sandra Laugier, « Emerson, père fondateur ? ", Raisons politiques, n²4, novembre 2006, p. 9-31. 
7. Ralph Waldo Emerson, Société et Solitude, Barcelone, Payot-Rivages, 2015, p.13.

8. Jacques Derrida, Donner la mort, Paris, Galilée, 1999, p. 85.

9. David Hume, Système sceptique et autres systèmes, Paris, Seuil, 2002, p.178-250.

10. Nous retrouvons mutatis mutandis un processus analogue à celui décrit dans la légende du Grand Inquisiteur dans Les Frères Karamazov. Ce personnage avoue à Jésus qu'il a suivi les « conseils » politiques du Tentateur (du diable, du double) des chapitres 4 des Évangiles de Luc et Matthieu, plutôt que ses enseignements politiquement inapplicables. Or cet aveu est ce qui perd irrévocablement l'Inquisiteur selon Ivan qui raconte l'histoire, quand, au contraire, c'est ce qui le sauve selon Alexeï qui reçoit le récit en embrassant son frère. Fiodor Dostoïevski, Les Frères Karamazov, Paris, Folio, p.345-368.

11. Jacques Derrida, Donner la mort, Paris, Galilée, 1999, p. 173-190.

12. Stanley Cavell, The World Viewed, Harvard, Harvard University Press, 1979, p. 16-23.

13. Au demeurant, Bazin ne soutenait lui-même pas autre chose lorsqu'il écrivait que la photographie telle qu'elle fut employée par les surréalistes et leurs collages magiques produisait des « hallucinations vraies ». André Bazin, Qu'est-ce que le cinéma ?, Paris, Cerf, 1999, p.9-17. Ce en quoi son réalisme rejoint sans paradoxe l'assertion de Mourlet : «Le cinéma est un regard qui se substitue au nôtre pour nous donner un monde accordé à nos désirs ", Michel Mourlet, L'Écran éblouissant, Paris, PUF, 2011, p. 201-205.

14. Briony Addey "Don't mistake coincidence for fate ", The Ultimate Lost and Philosophy: Think Together, Die Alone, éd. Sharon Kayes, Hoboken, John Wiley \& Sons Inc., 2011, p.91-119.

15. Erwin Panofsky, «Style et Matériau au cinéma » in Cinéma, Théorie, Lectures, éd. Dominique Noguez, Paris, Klincksieck, 1973, p. 58.

16. Ibid.

17. Ibid. C'est pour cette même raison qu'un réalisateur comme Robert Bresson va jusqu'à bannir le terme d' « acteur » oscillant entre l'idolâtrie de la star et l'anonymat du cliché et parlera plutôt de « modèle " pour insister sur le potentiel épiphanique du médium cinématographique.

18. Thomas d'Aquin, Somme Théologique, I, Paris, Cerf, 1984, p. 516-517.

19. Stanley Cavell, The World Viewed, Harvard, Harvard University Press, 1979, p. 35.

20. Ibid., p. 72.

21. Ibid., p. 68-73.

22. Ibid., p. 236-237.

23. Dédoublement qui est la perte du Monstre de fumée puisqu'en inspirant la profanation de Jacob à Benjamin, en apparaissant comme Locke, il a aussi inspiré Jack (Matthew Fox) qui en se sacrifiant peut reconnaître le vrai Locke, lorsqu'il affirme : "You're not John Locke. You disrespect his memory by wearing his face, but you're nothing like him. Turns out he was right about most everything. I just wish I could have told him that while he was still alive. » C'est-à-dire que Jack ne reconnaît pas en celui qui lui ressemble celui que Locke est et a toujours été.

24. Philippe Corcuff et Sandra Laugier. «Perfectionnisme démocratique et cinéma: pistes exploratoires ", Raisons politiques, vol. 38, $\mathrm{n}^{\circ} 2,2010$, p. 31-48.

25. Emerson, Société et Solitude., 2015, p. 18.

26. Précisons que Hurley trouve Crainte et Tremblement (en français dans le texte) dans le souterrain sous le mur du Temple dans l'épisode «LAX part 2 » (S06E02) en se demandant : «Who reads a book in a cave? ». Quant à Dostoïevski, il est explicitement cité dans l'épisode " Maternity leaves " (S02E15) lorsque Locke donne à lire à Ben/Henry captif, Les Frères Karamazov. Celui-ci préfèrerait lire du Stephen King, mais est très intéressé d'apprendre que Hemingway entretenait une admiration ambivalente pour le russe. Le motif du «souterrain » se retrouve dans la tirade de Dmitri, le fils présumé parricide en prison, qui est une reprise d'un motif développé dans Le Manuscrit du Souterrain du même Dostoïevski, qui était nous semblait-il une des sources de la saison 2 . 
27. Pacôme Thiellement, « Lost ou le Vade-mecum des malades d'amour », TV/Series, hors-séries n ${ }^{\circ} 1,2016$, http://tvseries.revues.org/1614.

28. Emerson, Société et Solitude, 2015, p. 16.

29. La différence entre le rapport « On/Ils» et la relation personnelle «Je/Tu », le primat du renversement de perspective, de la substitution à l'autre, par rapport aux questions du bonheur, du devoir voire de la liberté, est ainsi manifeste dans la communication décisive, au malentendu inévitable, entre Benjamin et Jacob. Benjamin ressassant son désarroi dans une logique de la représentation et de la comparaison avec et contre le double de Locke et Jacob, demandant « What about me? » alors que l'Autre répond: «What about you?» dans l'épisode « The Incident » (S05E17).

30. Il y a des personnages moins malades que d'autres : les plus évidents étant Vincent, le chien évoluant dans un temps pré-humain, porteur d'une mémoire pré-égocentrée, et qui est pourtant domestique, Aaron, le nouveau-né qui est tout en futur ; les personnages féminins, type «fille du diable » ou «fille de l'Ennemi », qui n'ont pas de flashback mais dont le rôle est déterminant comme Penelope (Sonia Walger) pour Desmond et pour Charles, et Alexandra pour Benjamin ; des personnages qui ont des flashbacks partagés comme Rose (Laverne Scott Caldwell) et Bernard (Sam Anderson) dans l'épisode "SOS» (S02E19) voire Jin (Daniel Dae Kim) et Sun (Sun-Hwa Kwon) qui sont toujours et ensemble et séparés (comme ces amants stellaires de la légende chinoise qui inspira à Claudel l'écriture du Soulier de Satin), et dont la fille Ji Yeon est le trait d'union à travers l'espace et le temps dans le très bel épisode "Ji Yeon » (S04E07). Jacob et sa «fille», Ilana, ne relèvent pas tout à fait de ce schéma non plus. Enfin on peut considérer que les personnages qui, dès à présent, voient les morts et l'avenir et/ou sont internés, comme Hugo, Libby (Cynthia Watros), Walt (Malcolm David Kelley), dans la mesure où ils souffrent explicitement, sont des témoignages vivants du mensonge d'un ego centre de flashback ou de flashforward.

31. Cette passivité fondamentale se prête mieux à un traitement sonore que visuel et se retrouve dans le fait qu'ils sont interpelés par le regard du téléspectateur et réciproquement, comme l'illustrent les très gros plans des ouvertures d'yeux, plans souvent précédés d'un son quand l'écran est noir, dans la plupart des épisodes de début de saison. Cette passivité, cette patience, est aussi le regard hors-champ qu'on devine après coup avoir été celui de Vincent qui ouvre et clôt la série en regardant l'ouverture et la fermeture de l'œil de Jack (Matthew Fox). Le chien est dans L'Odyssée la fidélité même, celui qui reconnaît Ulysse et dont la mort prépare à la reconnaissance par les hommes.

32. Cyril Béghin, « Faire une série », Cahiers du Cinéma, n735, Juillet/Août 2017, p. 14-15.

33. Emerson, La Confiance en soi, Barcelone, Payot-Rivages, 2000, p. 82.

34. Jean-Marie Schiff, L’Âge Cosmique aux U.S.A., Paris, Albin Michel, 1981, p.191.

35. Idem pour les téléspectateurs entre eux, l'interprétation produite donne à un téléspectateur raison contre et avec une interprétation concurrente.

36. Ralph Waldo Emerson, La Confiance en soi, Barcelone, Payot-Rivages, p. 82.

37. Sarah Hatchuel, Lost : Fiction vitale, Paris, PUF, 2013, p. 34-47. On se reportera aussi à l'article de Claire Cornillon dans cette même revue qui définit à juste titre Lost comme un « ensemble show " réclamant une expérience affective, l'amour du récit, de la part du téléspectateur en citant le « I'll have you » d'Ilana à la fin de l'épisode « Dr Linus » (S06E07) qui, nous semble-t-il, est d'autant plus fort que ce thème de la reconnaissance est mis en rapport avec Benjamin Linus/Michael Emerson personnage détestable auquel on finit par s'attacher à ce moment. Claire Cornillon, « Lost, fiction kaléidoscopique ", TV/Series, hors-séries n¹, 2016, http://tvseries.revues.org/1632.

38. Platon, Phèdre, Paris, Garnier-Flammarion, 1992, p. 117-120. Ralph Waldo Emerson, « Destin, la conduite de la vie " in Stanley Cavell, Statuts d'Emerson: Constitution, Philosophie, Politique, Combas, L'Eclat, 1992, p. 67-69.

39. Si l'on considère qu'il faisait partie de la famille Dharma. 
40. L'épisode «Catch-22» (S03E17) reprend le titre du roman de Heller, œuvre quasiment intégralement écrit selon le principe de la double contrainte et de l'effort pour rendre l'autre fou. 41. Paul Watzlawick, Faîtes vous-mêmes votre malheur, Paris, Point-Seuil, 2014.

42. Stanley Cavell, Conditions nobles et ignobles, Combas, L'Eclat, 1993, p. 66.

43. Là encore, l'homonymie aidant, et si l'on considère Hume le philosophe écossais, cet état d'esprit est sans doute la part du « wit », le tour de pensée qui rend l'homme aimable en société et créatif en cultivant les conséquences de pensées improbables par l'imagination dans Le Dialogue sur la religion naturelle. D'autre part ce personnage est lui-même double, Desmond étant l'homonyme d'un personnage du film Twin Peaks: Fire Walk with me (réal David Lynch, 1992), Chet Desmond (Chris Isaak), qui est le premier à évoquer la « rose bleue » de la série.

44. Stanley Cavell, Statuts d'Emerson: Constitution, Philosophie, Politique, Combas, L'Eclat, 1992, p. 9.

45. Dans quasiment tous les épisodes centrés sur lui, Locke répète d'une manière ou d'une autre qu'il est spécial, quand il ne dit pas «Don't tell me what $i$ can't do!». Comme ce dernier, dans l'épisode «Deus ex machina » (S01E19), la mère (Allison Janey) adoptive du jumeau de Jacob lui dit qu'il est spécial avant qu'il ne fasse sienne cette proposition contre la personne qui la lui a inspirée dans l'épisode « Accross the sea » (S06E15).

46. Pacôme Thiellement, La Main gauche de David Lynch, Paris, PUF, 2016, p. 18-53.

47. Au demeurant la lecture de Bad Twin, nous apprend que le purgatoire est clairement pensé par les auteurs comme l'espace de la fiction et de l'image, quand bien même s'agirait-il d'un purgatoire démystifié et traité ironiquement. Gary Troup, Bad Twin, Paris, Fleuve Noir, 2007, p. 126-130.

48. Dans l'épisode «The Long Con » (S02E13), Sawyer paraît expliquer à Cassidy (Kim Dickens) le principe d'une arnaque réussie : ne pas se cacher mais au contraire rendre visible un détail sur lequel viendront buter les observateurs, technique reprise dans l'épisode «B.J. and the A.C.» (S01E04) de The Leftovers scénarisé par le même Lindelof. Cette technique du «diable dans le détail » et corollairement de la dissimulation par l'évidence (comme dans La Lettre volée de Poe) serait comme une indication sur la manière de décoder l'écriture sérielle en distinguant l'accessoire de l'essentiel. Si ce n'est qu'en définitive Sawyer n'a appris à Cassidy à décoder une arnaque que pour l'arnaquer...

49. Ralph Waldo Emerson, La Confiance en soi et autres essais, Barcelone, Payot-Rivages, 2015, p. 129-135.

50. Maurice Maeterlinck, « Emerson » in Le Trésor des Humbles, Paris, Grasset, 2015, p. 95.

51. L'écriture n'est pas illogique mais lorgne souvent du côté de la rhétorique et de la poétique, d'où un discours à mi-chemin entre un commentaire voire une homélie profane sur le chapitre 22 de la Genèse, sur le chapitre 14 de l'Evangile de Luc et le Livre de Tobie une critique littéraire sur la tragédie d'Iphigénie, le conte d'Andersen Agnès et le Triton, sur Faust quelques allusions autobiographiques aux fiançailles avortées de l'auteur, le tout écrit sous le pseudonyme Johannes de Silentio, renvoyant au silence fervent abrahamique. Nous conjecturons que la version française de Crainte et Tremblement dans le sac d'un personnage dénommé Montand est un clin d'œil au Salaire de la peur (réal. Henri Georges Clouzot, 1953) dont le héros est incarné par Yves Montand. À moins que le choix d'un intitulé étranger à la langue de l'auteur scandinave et à la langue de la série ne soit une interprétation du silence fervent et de la manière d'en répondre dont Kierkegaard écrit : « Il ne professe ainsi nul mensonge, bien qu'il ne dise quoi que ce soit, car il parle en une langue étrangère. » Søren Kierkegaard, Crainte et Tremblement, Paris, PayotRivages, 1999, p. 202.

52. Stanley Cavell, Statuts d'Emerson., p. 26.

53. Troup, Bad Twin, p. 180.

54. Maurice Maeterlinck, « Emerson », in Le Trésor des Humbles, Paris, Grasset, 2015, p. 98.

55. Sarah Hatchuel et Randy Laist, «Lost: une « romance » shakespearienne ?», TV/Series, horsséries $\mathrm{n}^{\circ} 1,2016$, http://tvseries.revues.org/1656. 
56. Florent Favard, « "You can('t) let go now": Mystery boxes, quantum readings and very bright lights in Lost », TV/Series [En ligne], Hors séries 1|2016, mis en ligne le 01 octobre 2016: http:// tvseries.revues.org/1738

57. Walter Benjamin, Euvres III, Paris, Folio, 2001, p. 67-151 et p. 269-316.

58. Sur cette présence cachée révélée par l'image cinématographique comme reprise de l'inspiration travaillant la langue de Maeterlinck, on peut se reporter à la partie de l'essai d'Eugène Green, Présences, auquel l'intitulé de cette dernière partie rend hommage. Cet hommage est hérétique, toutefois, puisqu'il s'agit de transposer son économie du cinématographe dans celle de la télévision en étudiant, qui plus, est une série états-unienne donc «barbare » dans la terminologie du réalisateur. Pour autant la perspective d' " autres » États-Unis, spirituels traverse la pensée d'Ermerson et Lost, mais pour ce faire il faut en découvrir le fond de barbarie. Eugène Green, Présences : Essai sur la Nature du cinéma, Paris, Desclée de Brouwer/Cahiers du Cinéma, 2003, p. 103-127.

59. Maeterlinck, Le Trésor des himbles,Paris, Grasset, 2015, p. 182.

60. Stanley Cavell, Statuts d'Emerson, Combas, L'Eclat, 1993 p. 27.

\section{ABSTRACTS}

Lost is a polysemic show which mobilizes, for that purpose, the trope of homonymy. Many characters go by the name of great historical figures. This process questions the theme of utopia and ideal society. Yet Benjamin Linus, the main antagonist from the $2^{\text {nd }}$ season to the $5^{\text {th }}$, is impersonated by an actor named Michael Emerson, whose name is the same as the American philosopher, Ralph Waldo Emerson, on whom Cavell says that is one of the repressed founding father of American thought. Under what circumstance can we consider that this homonymy constitutes a reference to understand the fictional narrative without taking the risk of confusing our desires with reality? Our thesis is that Lost encourages this sort of risk-taking. The story is a narrative experience, in the wake of Emerson's self-titled essay. It incites us to rely on ourselves, to dare claim that each of us is special, in order to think a televised subjectivity and intimate recognition at odds with the egocentric voyeur's posture of the standard viewer.

Lost est une série polysémique qui mobilise, à cet effet, le trope de l'homonymie. Nombre de protagonistes portent le même patronyme que des personnalités historiques ce qui interroge le thème de l'utopie et de la société idéale. Or l'antagoniste principal constamment refoulé dans la série, de la saison 2 à la saison 5, Benjamin Linus, est "personnifié » par un acteur dénommé Michael Emerson, patronyme homonyme du philosophe américain Ralph Waldo Emerson dont Cavell dit qu'il est l'un des pères fondateurs refoulés de la pensée américaine. À quelle condition pouvons-nous considérer que cette homonymie réelle constitue une référence pour comprendre la narration fictive sans courir le risque de prendre nos désirs pour des réalités ? Notre thèse est que Lost encourage une telle prise de risque. Le récit est une expérience narrative, dans le sillage de l'essai éponyme d'Emerson. Il nous incite à nous faire confiance, oser affirmer que chacun d'entre nous est spécial, afin de penser une subjectivité télévisée et une intime reconnaissance, en rupture avec la posture du voyeur égocentré du téléspectateur standard. 


\section{INDEX}

Mots-clés: Incarnation, rédemption, confiance en soi, utopie, Lindelof Damon, Emerson Ralph Waldo, Cavell Stanley, Maeterlinck Maurice.

Keywords: Impersonation, redemption, self-reliance, utopia, Lindelof Damon, Emerson Ralph Waldo, Cavell Stanley, Maeterlinck Maurice

\section{AUTHOR}

\section{GUILLAUME DULONG}

Guillaume Dulong, agrégé de philosophie et docteur en études cinématographiques, est professeur de philosophie et de cinéma en lycée, ainsi que chargé de cours en cinéma à l'université Michel de Montaigne Bordeaux. S'inspirant des théories d'André Gaudreault et Tom Gunning, son travail de thèse a porté sur le genre cinématographique de l'imaginaire et l'influence des technologies numériques sur les modes de narration contemporains de fantasy. Guillaume Dulong, who holds an 'agrégation' in philosophy and a PhD in film studies, is a professor of philosophy and cinema in high school; he also teaches cinema at the University of Bordeaux Michel de Montaigne. Inspired by the theories of André Gaudreault and of Tom Gunning, his PhD thesis explored the filmic genre of fantasy and the influence of digital technologies on contemporary narration. 